\title{
As Reuniōes Técnicas como Fator de Integração e Atualização Profissional
}

Criadas para atender a uma necessidade de interação e troca de experiências dos profissionais de polímeros, as Comissões Técnicas da ABPol vêm cumprindo um papel importante ao longo dos anos, com resultados práticos evidentes, como no caso dos testes interlaboratoriais, além de transmitir conhecimentos, incorporados a todo um conjunto de procedimentos necessários para vários setores industriais. A presente matéria é uma breve abordagem sobre os objetivos, o trabalho realizado e os resultados alcançados pelas Comissões.

Dentro dos princípios da ABPol de valorização dos recursos humanos, encarados como instrumento básico de todo desenvolvimento, vem sendo realizado já há alguns anos um trabalho visando à integração e à troca de experiências entre os profissionais do setor, sejam do meio acadêmico ou industrial, gerentes, técnicos de laboratório, pesquisadores ou estudantes universitários.

O contato entre os diversos grupos tem ocorrido nas reuniões das Comissões Técnicas, cujos temas básicos são escolhidos de acordo com as necessidades da própria comunidade que as compõe e norteia. Surgindo espontaneamente ao longo do tempo, na medida em que a Associação se consolidava e passava a ser encarada como um prestador de serviço para o setor, foram sendo formadas essas Comissões, das quais a de Identificação e Caracterização de Polímeros é a mais antiga. Nos últimos dois anos foram criadas também as de Reciclagem de Polímeros e de Reologia e Processamento de Polímeros.

\section{Identificação e Caracterização de Polímeros}

As atividades da Comissão de Identificação e Caracterização de Polímeros se iniciaram em maio de 1992, sob a liderança do Prof. Sebastião V. Canevarolo Jr., então diretor da ABPol. Alguns meses depois os participantes elegeram como coordenador Wilson Franco (Plascar), que dirigiu as reuniões durante dois anos, sendo substituído por André Sautchúk (exPolimate) que, há dois anos e meio passou a coordenação para Manuel Mendes Filho (Telebrás).

O escopo principal dessa Comissão é a promoção do intercâmbio de conhecimentos e recursos materiais entre os profissionais, para resolução de problemas na área de identificação e caracterização de polímeros e seus produtos. Esses objetivos vêm sendo concretizados através da discussão de temas importantes durante as reuniões, além da realização de testes interlaboratoriais e encontros, como o workshop ocorrido em maio de 1998, em conjunto com a Comissão Técnica de Reciclagem, e que reuniu cerca de 100 participantes.

O interesse da comunidade pela Comissão de Caracterização levou à realização de 46 reuniões, 27 palestras técnicas e 13 testes interlaboratoriais já concluídos e outros dois em andamento. Acrescente-se ainda dois workshops e um seminário. A participação da comunidade também tem sido das mais abrangentes, pela presença nas reuniões de representantes de empresas e instituições como Arno, Bayer, Branco, Brancolor Cromex, CCDM/UFSCar, CEPED, CEPEL, Ciba, Cofade, CTA/IAE, Enxuta, EFEI, Esc. Técnica Tupy, Fairway, G.E. Plastics, Hoechst, IPEN, Ipiranga Petroq., Johnson, Laboratórios B. Braun, Petroq. Triunfo, Pirelli Cabos, Plascar, Plást. Maradei, Plást. Mueller, Polialden, Policarbonatos, Polo, Resana, Robert Bosch, Scania, Senai Mário Amato, Senai/Cetind, Sogefi, Telebrás, Ticona, Unicamp, Union Carbide, Universidades diversas (UFRGS, UCS, UFPI, USP, 
Unisc, etc.), VCP - Votocel, Volkswagen, etc.

O atual coordenador da Comissão, Manuel Mendes Filho, tem observado que, mesmo tendo grande interesse nas palestras técnicas, a atenção das empresas tem-se centralizado nos testes interlaboratoriais, o que é perfeitamente compreensível, uma vez que eles abrangem diferentes propriedades dos materiais poliméricos, como impacto, índice de fluidez, tração, análise térmica por DSC, tempo de indução oxidativa(OIT), espectrofotometria na região do infravermelho e, mais recentemente, análise térmica por TGA e DMA. Afinal, como as empresas podem garantir a qualidade dos produtos e, conseqüentemente sua competitividade no mercado sem que, internamente, tenham uma garantia mínima do que estão produzindo? Se os materiais devem ser testados, como fazer para calibrar os equipamentos sem ter que contratar pessoal especializado e sem despender recursos internos que estão cada dia mais reduzidos? A resposta a essa pergunta pode ser fácil e consistente se houver a participação nos testes interlaboratoriais.

E o que eles vêm a ser exatamente? Utilizando um equipamento devidamente calibrado, a coordenação do teste faz a análise prévia de algumas amostras do material que será distribuído para os demais participantes do interlaboratorial. Além desse teste ser feito com o máximo de rigor, o material a ser utilizado é de um único lote e suas especificações já são conhecidas, de forma a se saber tanto o que está sendo analisado como a faixa aceitável de variação dos resultados. Após essa etapa, todos os participantes recebem os corpos de prova e as normas para a realização do

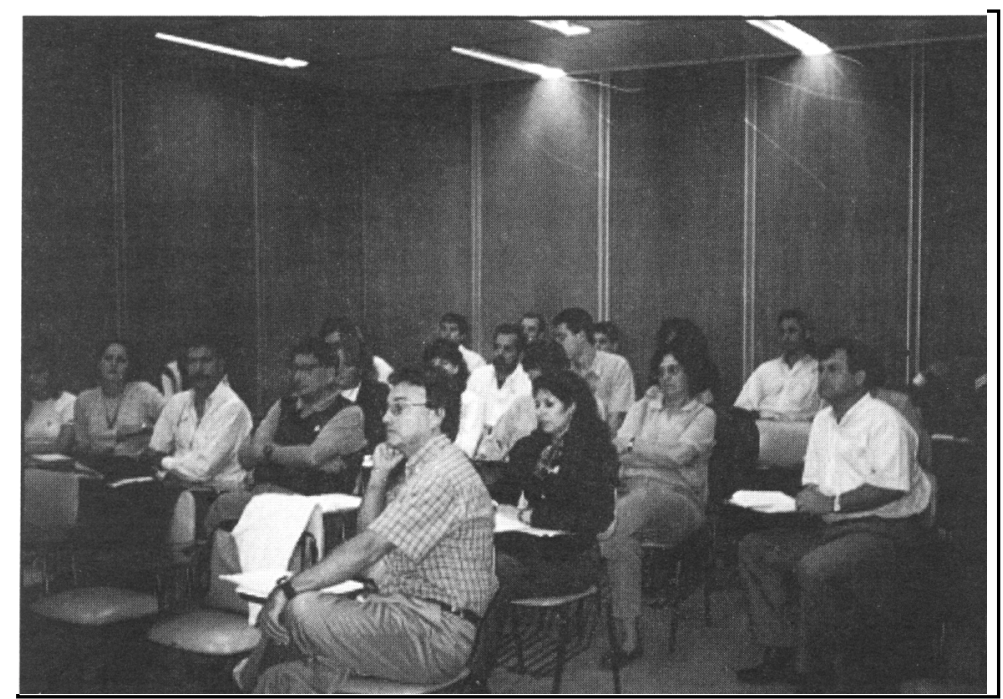

Reunião da Comissão Técnica de Identificação e Caracterização de Polímeros ocorrida em 18 de março passado.

teste. Na etapa seguinte é feita a avaliação dos resultados obtidos, de acordo com a faixa de variação permitida. Cada participante recebe informações detalhadas sobre seus resultados e seu posicionamento em relação às empresas do setor, tendo, então, condições de verificar a consistência e confiabilidade dos resultados gerados pelos seus laboratórios. Todavia, é exatamente na etapa de tratamento estatístico dos resultados dos testes interlaboratoriais que tem ficado evidente uma certa falta de conhecimento dos métodos de ensaio adotados e até um certo despreparo dos técnicos/ operadores, ou seja, de quem está fazendo o teste e mesmo supervisionando a apresentação dos resultados nos protocolos de ensaio encaminhados aos coordenadores dos testes para avaliação. Assim sendo, a não-calibração dos equipamentos acaba sendo mais um dos vários problemas sérios observados na análise dos resultados apresentados pelos laboratórios participantes. As deficiências de conhecimento técnico à respeito podem ser amenizadas através da participação de funcionários da empresa na Comissão.
É importante se ter em mente também que, se a maioria dos profissionais não tem condições de freqüentar cursos regulares de aperfeiçoamento e nem a empresa tem condições de liberar um funcionário para isso, a participação em reuniões técnicas como as das Comissões é uma opção eficiente e barata de aprendizagem informal.

Quanto às discussões técnicas, apenas para exemplificar sua diversidade e a relevância dos temas, segue a relação dos títulos das apresentações de 1997, feitas nas reuniões da Comissão de Identificação e Caracterização de Polímeros:

- "Análise dos Resultados do Interlaboratorial de DSC" - Selma B. Jaconis - IPT

- "Análise dos Resultados do Interlaboratorial de O.I.T." - Selma B. Jaconis, IPT

- "Espectroscopia no Infravermelho aplicada a Polímeros" Yoshio Kawano, USP/IQ

- "Tratamento Corona em Filmes e Peças Plásticas" - Edson da Cunha Almeida, Inductoheat Ind. e Com. Ltda.

- "Caracterização de Antioxidantes em Poliolefinas por HPLC"- 


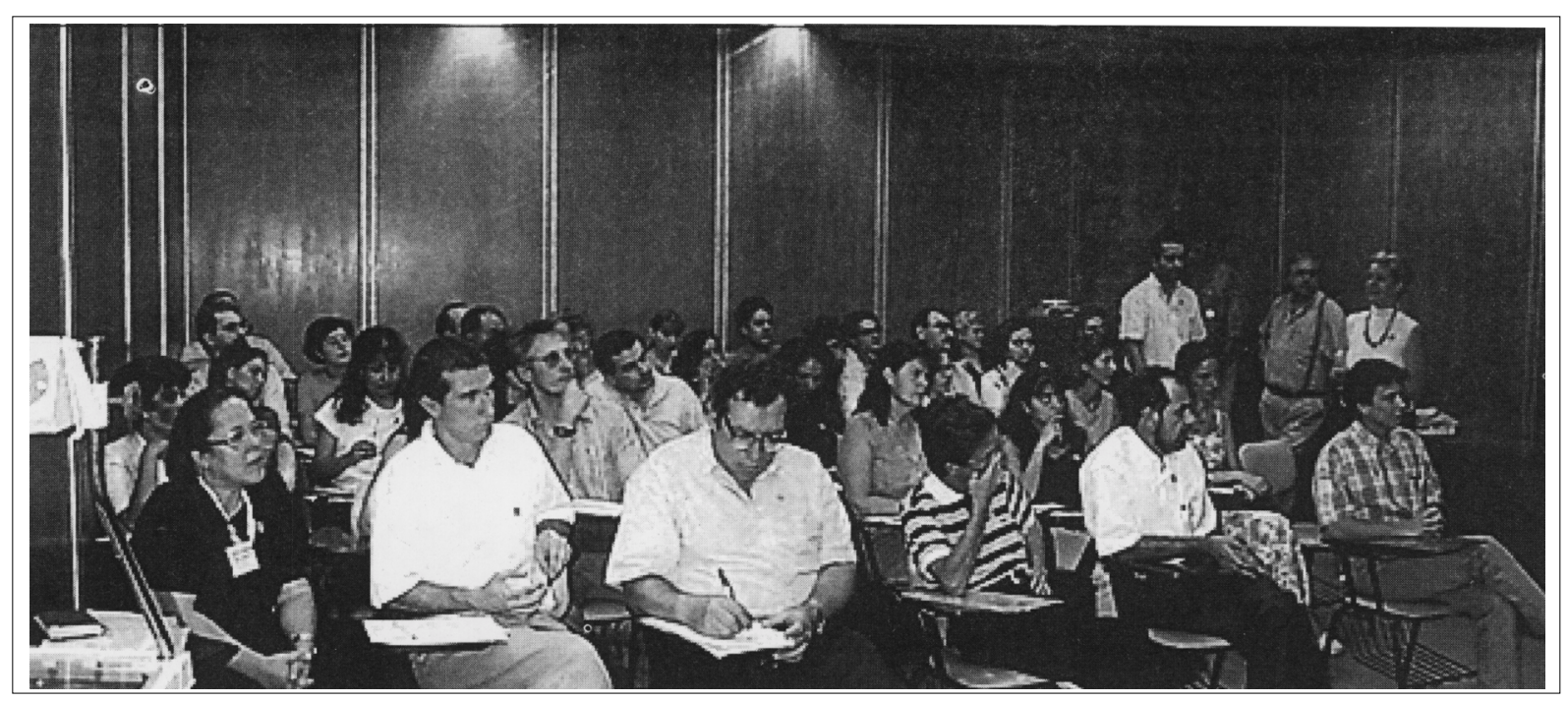

Reunião da Comissão Técnica de Reciclagem de Polímeros ocorrida em 4 de março passado

Sirney Silveira, $\mathrm{CPqD} /$ Telebrás

- "Análise de Falhas em Materiais através de Técnicas Avançadas de Microscopia" - Carlos Alberto Correa - CCDM/UFSCar

- "Apresentação da Análise Estatística dos Resultados do $3^{\circ}$ Interlaboratorial para aferição de Espectrômetro Infravermelho" Selma Barbosa Jaconis, IPT

- "As Novas Tendências do Ensaio de Intemperismo Artificial no Controle de Materiais Poliméricos"Carlos Alberto Fazano - Panambra Indl. e Técnica S/A.

- "Avaliação do Desempenho de Materiais Poliméricos através da Técnica de Múltipla Reflectância" - Fabio Noronha - Telebrás/CPqD

\section{Reciclagem de Polímeros}

Em meados de 1996 foi criada a Comissão Técnica de Reciclagem de Polímeros, a exemplo da Comissão de Identificação e Caracterização de Polímeros, que já dava bons frutos e conseguia destaque na comunidade acadêmica e dentro do parque industrial de polímeros. A área de reciclagem ganhava também grande importância na área de resinas, tanto pelo volume de material reciclado como pelo surgimento de novas tecnologias e de equipamentos importados sofisticados. O Prof. Hélio Wiebeck, da Escola Politécnica da USP aceitou, então, o desafio de coordenar a nova Comissão e mobilizar a comunidade para que participasse das reuniões.

A comunidade acadêmica preocupava-se de modo mais acentuado com a reciclagem de resíduos, porém o surgimento da ISO 14000 despertou o interesse das empresas, principalmente por causa da responsabilidade do resíduo gerado. A Comissão de Reciclagem se propôs, na verdade, a criar um fórum de discussão que viabilizasse: 1- a troca de experiências e de conhecimento; 2- a interação entre universidades, empresas, centros de pesquisa e entidades diversas; 3- a promoção da viabilidade da reciclagem no país, quanto a aumento de volume, melhora de tecnologia e redução de impostos.

A resposta dos profissionais da área veio prontamente com a participação, nas 13 reuniões realizadas, de recicladores em geral, fabricantes de equipamentos, professores, alunos, pesquisadores, fornecedores de resinas e aditivos, industriais, ecologistas, etc., oriundos de empresas e instituições como Abivinila, Abremplast, Aviv Tecnologia, Basf, Brampac, CAPP, Ciba, Clariant, Coveg, Cromex, Desfio, Engepack, Fairway, GMB, IMA/ UFRJ, Instituto do PVC, Mariplast, Metalúrgica Ricardo, Miotto, USP, Pallman, Pet Way, Petronyl, Plást. Maradei, Plastital, Plastivida, Polibrás, Polibrasil, Polilab, Poliset, Polymerpar, Prealpina, Raitek, Resinac, Rhodia, Roscaplas, Senai Mário Amato, Telebrás, UFSCar, Unesp, Unicamp, Unicastelo, Uniroyal, Wortex, etc.

Próxima a completar 2 anos de atividade, a Comissão vem contando com a participação de um número maior de pessoas, reflexo de sua penetração junto aos profissionais da área. O nível das palestras apresentadas nas reuniões; a ajuda prestada a recicladores em questões técnicas; o sucesso do workshop realizado em 1997, em conjunto com a Comissão de Caracterização; a parceria com a Plastivida para a realização de um novo seminário em maio próximo, tudo isso são realizações que vêm consolidando a Comissão.

Segundo o Prof. Wiebeck, os maiores esforços da Comissão de Reciclagem têm sido direcionados 
para: 1- promover uma participação maior de recicladores de plástico; 2 o encaminhamento ao Governo de um documento pleiteando redução de impostos para os reciclados; 3promover uma maior interação entre outras associações ou entidades ligadas à área de polímeros.

Uma das últimas propostas do grupo é a confecção de um manual para auxiliar os recicladores que, na maioria das vezes, não têm como conseguir informações técnicas básicas para constituir uma empresa de reciclagem. Sem esse conhecimento mínimo, os reciclados perdem qualidade, as empresas não sobrevivem e a imagem da reciclagem em si pode ficar comprometida, o que é inaceitável quando se sabe que o aumento assustador de resíduos sólidos tem consumido áreas e mais áreas para sua disposição, e a reciclagem pode minimizar isto.

Segue a relação das palestras apresentadas durante o último ano:

- "Atividades da Abremplast" - Liviu Schwarz (Abremplast)

- "Reciclagem de PET" - Paulo R. Straiotto (Pet Way Reciclagem Ltda.)

- "Reciclagem de PET" Vladimir Kudrjawzew (Repet)

- "Injeção de PET" - Sandro

D. Mancini (UFSCar)

- "Linhas de Pesquisa em Reciclagem na UFSCar" - Sati Manrich (UFSCar/DEMa)

- "Controle de Qualidade de Materiais Reciclados para Peças Técnicas (Plásticos de Engenharia)" - Marlos G. de Oliveira (Petronyl)

- "Aditivação em Plásticos Reciclados" - Antonio Rodolfo Jr. (Itap-Cromex)

- "Dificuldades da Reciclagem de PVC" - Eduardo V. Silva (CCAPP) e Carlos Alberto V. Azevedo (Desfil), recicladores de PVC

- "Como os Produtores de Re- sina de PVC Identificam a Reciclagem (Mito e Realidade)" - Alfredo Franchi (Abivinila)

- "Tecnologia na Reciclagem de PVC (Máquinas e Processos)" - Eduardo V. Silva (CCAPP)

- "Seleção de Roscas para Processos de Reciclagem"- Paolo de Filippis (Wortex)

- "Moagem"- Ralph C. Thieme (Palmann do Brasil)

\section{Reologia e Processamento de Polímeros}

A Comissão Técnica de Reologia e Processamento de Polímeros, coordenada pelo Eng. Júlio Harada, diretor de eventos da ABPol, surgiu no final de julho de 1997, em função da solicitação dos sócios em uma pesquisa realizada sobre as necessidades que a comunidade tem na área de processamento e transformação dos polímeros e também após o I Seminário Brasileiro de Avanços em Processamento de Polímeros, promovido em março do mesmo ano, pelo Núcleo de Excelência em Reologia e Processamento de Polímeros, da Universidade Federal de São Carlos, com mais de 250 participantes.

O objetivo é organizar reuniões que atendam as necessidades da comunidade, através da: 1 - reunião dos mais diferentes processadores de plásticos para discussão dos problemas do dia a dia; 2 - apresentação de novos produtos e novos conceitos para transformação de polímeros; 3 apresentação de trabalhos pelas mais diferentes personalidades da área. Os profissionais visados são os engenheiros, técnicos, gerentes, supervisores de processo e produção, tecnologia e qualidade das empresas de transformação de plásticos, o que se tem conseguido nas 5 reuniões já realizadas, com a participação de representantes de empresas e instituições como Basf,
Bayer, Capa-Centro, Dacarto, DEMa/UFSCar, Dinateste, Dow, EPUSP, Fairway, G.E. Plastics, GMB, ICP, Kanaflex, Ludovico, Mixcim, Pirelli Pneus e Pirelli Cabos, Plást. Maradei, Polibrasil, Rhodia, Romi, Senai Mário Amato, Semeraro, Telebrás, Viskase, Zanettini, etc.

Pelo pouco tempo que tem de atividade, a Comissão já pode contabilizar alguns bons resultados, como o excelente nível das palestras apresentadas e a realização de um seminário de dois dias, em conjunto com as demais Comissões, no final de maio próximo. Para esse evento, além de vários palestrantes nacionais, haverá a apresentação de um "short course" sobre Automatização em moldagem por injeção, ministrado por Lance Neward, especialista da SPE. Quanto aos planos para o futuro, o Eng. Júlio Harada acredita que, com uma maior participação da comunidade, há forte tendência a se formarem grupos de trabalho para atender à comunidade de determinados processos de transformação.

Durante s reuniões realizadas no ano passado, foram apresentadas as seguintes palestras:

- "Otimização de Processamento na Fabricação de Compostos" - José Alexandrino de Sousa (UFSCar/DEMa)

- "Variação de Fluidez e os Problemas de Processamento do Elastollan" - Cirenini Aprileo (Cofade)

- "Evolução do Processo de Injeção com o Auxílio de um Gás Inerte Pressurizado" - Ivan Braga Ramos (Indústrias Romi)

- "Tecnologia CAE para Injeção dePlásticos"--NG Kin Pui (Camanho \& Consultores Associados)

- "New Injection Molding Inline Approach. Thermorheological Study of Thermoplastic Polymers at 
High Shear Rates" - G. Villoutreix (CNAM - Paris)

- "Otimização de Processamento de Polímeros e Projetos de Roscas Através de Simulação" - Glauco Ricardo de Moraes (Mixcim)

- "Plásticos de Engenharia da Basf" - Júlio Harada (Basf)

Identificação e Caracterização, Processamento ou Recicla- gem de Polímeros se resumem, afinal, em temas essenciais para discussão de problemas e as três Comissões Técnicas da ABPol nada mais são do que fóruns de discussão, onde os tópicos de interesse são definidos pelos próprios participantes. Como problemas e soluções devem ser as duas faces de uma única moeda, a interação entre os profissionais de diferentes empresas e instituições se transforma em mecanismo de aprendizagem informal, ao mesmo tempo em que propicia inequívocos benefícios em termos de qualidade e produtividade, cujo custo é tão-somente o da participação nas reuniões.

\begin{tabular}{|lcc|}
\hline \multicolumn{3}{|c|}{ Calendário das reuniões das Comissões Técnicas em 1998} \\
Identificação e Caracterização & Reologia e Processamento & Reciclagem \\
29 de abril & 26 e 27 de maio (seminário) & 15 de abril \\
29 de maio (seminário) & $1^{\circ}$ de julho & 28 de maio (seminário) \\
24 de junho & 19 de agosto & 29 de julho \\
26 de agosto & 30 de setembro & 9 de setembro \\
7 de outubro & 4 de novembro & 21 de outubro \\
18 de novembro & 16 de dezembro & 2 de dezembro \\
\hline
\end{tabular}

